\title{
Human Resource Petri Net Allocation Model Based on Artificial Intelligence and Neural Network
}

\author{
Weihuang Dai $\mathbb{D},,^{1,2}$ Yi Hu $\mathbb{D}^{1,3}$ Zijiang Zhu $\mathbb{D}^{1,3}$ and Xiaofang Liao $\mathbb{D}^{1,3}$ \\ ${ }^{1}$ Institute of Intelligent Information Processing, South China Business College, Guangdong University of Foreign Studies, \\ Guangzhou 510545, Guangdong, China \\ ${ }^{2}$ School of Management, South China Business College, Guangdong University of Foreign Studies, Guangzhou 510545, \\ Guangdong, China \\ ${ }^{3}$ School of Information Science and Technology, South China Business College, Guangdong University of Foreign Studies, \\ Guangzhou 510545, Guangdong, China
}

Correspondence should be addressed to Yi Hu; 201007@gwng.edu.cn

Received 12 June 2021; Revised 8 July 2021; Accepted 14 August 2021; Published 28 August 2021

Academic Editor: Sang-Bing Tsai

Copyright (C) 2021 Weihuang Dai et al. This is an open access article distributed under the Creative Commons Attribution License, which permits unrestricted use, distribution, and reproduction in any medium, provided the original work is properly cited.

The reasonable allocation and use of human resources is an important content in the process of complex system analysis and design. This paper studies the human resource allocation model of Petri net based on artificial intelligence and neural network. In this paper, combined with the characteristics of human resource scheduling, human resource mobility, concurrency, and obvious classification characteristics, the human resource allocation model based on Petri net is implemented. In this paper, the model is trained with the python version of human resource analysis data set. The training parameters are 100, the error coefficient is 0.001 , and the learning speed is 0.01 . First, the coding rules of human resource data are established. Then, the parameters are input into the model, and the human resource data are trained in the model. Finally, the results of the model output layer are analyzed. The research study shows that the average prediction accuracy of this model is $78.85 \%$. Model training requires the addition of 25 neurons for every 0.01 increase to improve the accuracy of predicting dynamic data of human resources. If the accuracy rate exceeds 75\%, the increase in the number of neurons cannot be compensated for by the increase in the accuracy rate, but it is most efficient when the amount of data for human resource scheduling is 2000 to 4000 . Therefore, this system can effectively allocate small- and medium-sized human resources and has a high accuracy.

\section{Introduction}

At present, China's structural reform of human resources is in a critical period, and the role of product innovation in the competitive market and economic reform is gradually highlighted. It has become an important path choice for enterprises to obtain leapfrog development, sustainable development, high-quality development, and market competitive advantage. What kind of strategic human resource management (SHRM) measures enterprises adopt to support and promote product innovation, in order to obtain sufficient development power and clear development goals, is one of the hot spots of management practice and theoretical research. Existing studies have confirmed that the effective SHRM system can help enterprises quickly gain market competitive advantage, and the SHRM research can be summarized into two views: configuration view and contingency view. Among them, the configuration view emphasizes the consistent combination within the management practice, and the contingency view emphasizes the vertical fit between the human resource practice and the external situational elements.

In order to solve the task scheduling problem in the cluster with limited manpower supply, Mom and others proposed a task scheduling method based on task value [1]. Salas vallina first predicted the running time of all tasks to be executed under all possible resource allocation conditions and then calculated the value of each task under each resource allocation condition [2]. The algorithm selects the most valuable task and provides the optimal cluster 
configuration for the task. Parallel tasks in heterogeneous clusters will compete for resources in the cluster, which will lead to the degradation of cluster performance. Prieto pastor proposed a two-stage task scheduling method based on reinforcement learning and neural collaborative filtering, which can solve the above problems through task segmentation [3]. Its learning driven load parallelization algorithm can find suitable running nodes for independent tasks. Gibson's spark resource scheduling method focuses on the reasonable allocation of computing resources but does not consider the network resources, which may increase the network delay and reduce the cluster performance [4]. Ahammad et al. used Petri net to model the locomotive scheduling process in detail, analyzed its deadlock free scheduling, and proposed the optimal scheduling strategy but did not further combine the scheduling strategy with the scheduling system to solve the locomotive scheduling automation problem [5].

On the structure optimization of Petri net, Kang analyzed the characteristics of the so-called hybrid workshop problem and proposed an improved genetic algorithm to maximize the efficiency of hybrid workshop planning and provide a solution for improving the efficiency of underground locomotive scheduling [6]. In 2020, park established a Petri net model for railway dispatching. In view of the principle of limited resources, few organizations can take into account many human resource practices at the same time and put it in an equally important position [7]. In order to make up for the deficiency of this theory, Jørgensen and Becker proposed that the combination of the Petri model and human resources can more deeply analyze the collaborative combination effect of multiple human resources practices [8]. The most direct is that Chang's research found that the good state of work drive and work enjoyment directly stimulates the dual behavior of employees, which largely reflects the connotation characteristics of human resource allocation [9]. Diaz-Fernandez has proposed indicators of human resource intermediation, capacity limitations, and the importance of corporate bachelor's degrees. This paper introduces the contribution of maximum flow transmission. This overcomes the drawback that traditional neutrality indices only consider power flow transmission along the shortest path between buses [10]. However, the existing research has not been able to analyze the relative importance of human resource internal practice from the Petri model, that is, when and what human resource management practice plays a core role in the acquisition of competitive advantage. Therefore, the primary purpose of this study is to clarify the relative importance of human resource internal practice. On the basis of configuration view and from the perspective of weight change, the situational effect of human resources is mainly manifested in two paths: resource-based and dynamic capability.

This paper studies the human resource allocation model of Petri net based on artificial intelligence and neural network. In this paper, combined with the characteristics of human resource scheduling, human resource mobility, concurrency, and obvious classification characteristics, the human resource allocation model based on Petri net is implemented. In this paper, the model is trained with the python version of human resource analysis data set. The training parameters are 100 , the error coefficient is 0.001 , and the learning speed is 0.01 . First, the coding rules of human resource data are established. Then, the parameters are input into the model, and the human resource data are trained in the model. Finally, the results of the model output layer are analyzed.

\section{Human Resource Allocation Factors and Petri Net Model}

2.1. Human Resource Allocation Factors. Human resource allocation, also known as flow at work, is used to describe this cognitive state in the text. A path of "enjoy with heart, create with great concentration" is used to stimulate Petri human resource allocation, including the interactive effect of testing ability and motivation-oriented Petri orientation, the mediating effect of human resource allocation sense, and the moderating effect of opportunity-oriented Petri orientation [11]. As motivation scholars have emphasized, intrinsic motivation is more durable than extrinsic motivation. The Petri human resource allocation system that influences employees' behavior by stimulating intrinsic motivation is not only more long-term than other paths but also the general trend of the era of humanistic management [12]. Individual duality is one of the frontier research studies of duality theory. Scholars define it as the multidimensional behavior level of employees who carry out exploration and development work at the same time. In the field of innovation, it is called individual Petri human resource allocation. Exploratory innovation focuses on searching for new knowledge, discovering new opportunities, and developing new technologies, while exploitative innovation is committed to further consolidating and improving existing capabilities [13]. As the driving force of enterprise innovation and development, if $\mathrm{R} \& \mathrm{D}$ and innovation personnel can treat conventional and unconventional work equally and carry out creative and continuous innovation activities in a balanced way, it will be of great help to the common realization of short-term and long-term goals of enterprises [14]. However, it is not easy to engage in exploratory and exploitative innovation activities at the same time, and it is difficult to avoid the conflict between cognition and resources, which makes many innovators "hard pressed" in the allocation of resources. In addition, both exploration and development have the function of self reinforcement. The former will induce employees to find it difficult to extricate themselves from the cycle of trying new things and failure, while the latter will lead to path dependence [15]. To overcome the above difficulties, some scholars say that innovators have a strong cognitive quality, actively change their thinking, and minimize the negative emotions caused by role conflicts and their condition. He pointed out that the greatest potential needs to be stimulated. So, what mechanisms can be used to stimulate the potential of innovative talents? For example, to improve Petri's ability to allocate talents. Organization level and individual 
level $\times$ Interaction effect box refers to the circle of the Petri human resource allocation system, which is easy to show the ability and motivation-oriented interaction effect. $X$ refers to the interactive product force, which has become the current research hot spot [16]. In many organizational mechanisms, top-down Petri-oriented mechanism undoubtedly occupies an important position. Petri-oriented mechanism always needs to stimulate individual cognitive organism (such as motivation) to influence their behavior, and Petri human resource allocation, a Petri-oriented mechanism characterized by high employee engagement, shows this effect [17]. Petri human resource allocation can stimulate employees' potential by improving their control and decision-making power over their own work and absorb employees' potential wisdom, ideas, and motivation to inject them into organizational performance [18]. The Petri human resource allocation system mainly includes three modules: personnel flow, evaluation and reward, and labor relations [19]. Scholars have mapped out three kinds of incentive orientation, motivation, and opportunity orientation (AMO framework) for these modules. Ability-oriented Petri-oriented mainly expands the skills, knowledge, and experience of employees by means of selection and training; motivation-oriented Petri-oriented mainly uses the reward and punishment system to induce employees to engage in the activities expected by the organization; opportunity-oriented and Petri-oriented give employees more room to play through authorization, cooperation, and other ways [20]. It is not hard to imagine that the Petri human resource allocation system based on AMO can help to improve employees' skills, motivation, and autonomy, and these abilities are the key quality basis for employees to engage in Petri human resource allocation [21].

2.2. Petri Net Model and Function Algorithm. Petri net has many elements, such as place and transition:

$$
P=\{p 1, p 2, \ldots, p n\} \text {. }
$$

The transmitter and receiver must exist in pairs, and the driver can be directly connected to the relevant device without conflict. Transition is divided into instantaneous change and time change. Like the original PN, temporal transition takes a certain amount of time to process:

$$
\begin{aligned}
\mathrm{PN} & =\frac{n \sum_{i=1}^{n} \sum_{j=1}^{n} w_{i j}\left(x_{i}-\bar{x}\right)(x j-\bar{x})}{\sum_{i=1}^{n} \sum_{j=1}^{n} w_{i j}(x i-\bar{x})^{2}} \\
& =\frac{n \sum_{i=1}^{n} \sum_{i \neq j}^{n} w_{i j}\left(x_{i}-\bar{x}\right)(x j-\bar{x})}{S^{2} \sum_{i=1}^{n} \sum_{j=1}^{n} w_{i j}}, \\
\text { transition } & =\frac{\sum_{j=1}^{k} \sum_{h=1}^{k} \sum_{t=1}^{n_{j}} \sum_{r=1}^{n_{h}}\left|y_{i j}-y_{h r}\right|}{2 n^{2} u} .
\end{aligned}
$$

Program statements are generally composed of data processing operations, while the functional behavior of program code is represented by the conversion process of data object type and number. In this paper, data objects of operations are regarded as resources, and program operations are events that lead to resource flow [22]. According to the above Petri net definition, the types and statements of data objects can be represented by places and transitions, and the number of data objects can be represented by directed arc weights:

$$
\operatorname{Petri}_{j h}=\frac{\sum_{Z=1}^{h_{j}} \sum_{r=1}^{n_{h}}\left|y_{j i}-y_{h r}\right|}{n_{j} n_{h}\left(u_{j}+u_{h}\right)}
$$

The code behavior process based on Petri net is described as follows: the type conversion relationship between data objects in a statement is represented by the direction of directed arc in Petri net, and the number of different types of data objects in a statement is represented by the weight of directed arc:

$$
\mathrm{P}_{\mathrm{ssim}}=1-\frac{\left(2 \mu_{x} \mu_{y}+C_{1}\right)\left(2 \sigma_{x y}+C_{2}\right)}{\left(\mu_{x}^{2}+\mu_{y}^{2}+C_{1}\right)\left(\sigma_{x}^{2}+\sigma_{y}^{2}+C_{2}\right)} .
$$

This section presents a modeling method of code behavior process based on Petri net. First, the code behavior modeling mechanism based on Petri net is clarified, and the corresponding relationship between Petri net elements and code elements is formulated:

$$
\begin{aligned}
O\left(d_{i}, w_{j}\right) & =P\left(d_{i}\right) P\left(w_{j} \mid d_{i}\right) ; P\left(w_{j} \mid d_{i}\right) \\
& =\sum_{k=1}^{K} P\left(w_{j} \mid z_{k}\right) P\left(z_{k} \mid d_{i}\right), \\
I_{\kappa} & =\frac{2 k}{k+1}+\left[\frac{1}{2}+\frac{1}{2 k}\right]\left[\frac{c_{2}-c_{1}}{3}\right]^{2}+\frac{2\left(c_{2}-c_{1}\right)}{3} .
\end{aligned}
$$

Based on the corresponding relationship, the static analysis technology is used to analyze and process the program code to complete the transformation from code to Petri net:

$$
C_{j h}=\int_{0}^{\infty} \mathrm{d} F_{j}(y) \int_{0}^{y}(y-x) \mathrm{d} F_{h}(x) .
$$

A single program statement includes related operation, input data object set processed, and output data object generated after processing [23]. For example, a function call statement is composed of a function name, a caller calling the function, a callee as a parameter, and a return data object. The function name is an operator, the calling and called data objects are input data objects, and the return data object is output data object. Program statements are triples:

$$
\begin{gathered}
\ln \left(\frac{P_{i t}}{P_{i t}-1}\right)=\alpha+\beta \ln P_{i t}-1+\text { caller, } \\
\text { callee }=\sum_{x=1}^{\theta} V x * I=\frac{\sum_{x=1}^{\vartheta}\left(\left(W+\mathrm{Si} / \sum_{1}^{n} W_{\mathfrak{\Im}}\right) / C+S+\mathrm{Sc}\right)}{\alpha+\beta \ln P_{i t}+O / C} .
\end{gathered}
$$


SC represents the set of operators, Si represents the set of input data objects, and $O$ represents the set of output data objects. For $\forall \mathrm{C} \in \mathrm{SC}$, the statement input data object $I$ corresponds to the input data object of operation $C$ and the statement output data object $o$ corresponds to the output data object of operation $C$ with the lowest priority, such as follows:

$$
\begin{gathered}
\ln \operatorname{Petri}_{i t}=a_{0}+a_{1} \mathrm{~d} u * \mathrm{~d} t+\sum_{i=1}^{N} b_{j} X u+\varepsilon_{u}, \\
f(\mathrm{a})=\frac{1}{N h} \sum_{i=1}^{N} k\left(\frac{X_{i}-x}{h}\right) .
\end{gathered}
$$

Obviously, the function of the whole statement should be reflected in the final result of the assignment operation, so the variable $a$ is taken as the final output object. The change process of the number and type of data objects can be simply summarized as follows: processing data objects through statements and converting a certain number of input data objects of specific types into output data objects of specific types [24]. The process can be described in Petri net as follows: through the direction and weight of input/output arc of statement transition, the transformation process from input data object to output object is represented.

$$
\begin{aligned}
\frac{\sigma t}{S} & =\frac{\sqrt{(1 / n) \sum_{i=1}^{n}\left(\mathrm{Si}_{i t}-\mathrm{Si}_{i t}\right)^{2}}}{\mathrm{Si}_{i t}+\mathrm{ts}}, \\
u_{(j \mid i)} & =\frac{w_{i j} A_{i}}{\left(\mathrm{Si}_{i t}-\mathrm{Si}_{i t}\right)} .
\end{aligned}
$$

The arc points from the input data object type to the output data object type and the weight of the input and output arc, respectively, represent the number of input data objects consumed and output data objects generated in the conversion process. For the statement $s \in s$, TS is used to represent the statement transition. For the input data object type $I \in \mathrm{Si}$, PI is used to represent the input data object type library. For the output data object type $O$, po is used to represent the output data object type library:

$$
\text { po }(x)=\frac{1}{\sqrt{2 \pi}} W(\text { pi, ts })\left(-\frac{x^{2}}{2}\right) .
$$

For the convenience of description, the input arc connecting statement transition and input data object type library is expressed as (PI, TS), and the weight of input arc is expressed as $w$ (PI, TS), that is, the number of input data objects of type $I$ in statement $s$. The output arc connecting the statement transition and the output data object type is expressed as (TS, PO), and the output arc weight is expressed as $w$ (TS, PO), and $w(\mathrm{TS}, \mathrm{PO})=1$. In Petri net, the consumption of input data objects and the generation of output data objects are used to model program statements, which is prone to inconsistency of code semantic description $[25,26]$. For example, for an active object $x$ that needs to be called many times, when $x$ executes a method call, it consumes the data object $x$ in the statement description of the corresponding Petri net, resulting in the inaccurate description of other program statements involving $x$ call in the future.

$$
\begin{aligned}
G_{t}(W(\mathrm{pi}, \mathrm{ts})) & =\sum_{j=2}^{k} \sum_{h=1}^{j-1} G_{j h}\left(p_{j} s_{h}+p_{h} s_{j}\right) D_{j h}\left(1-D_{j h}\right), \\
D_{H} & =\frac{p_{2}-p_{1}+1}{2} .
\end{aligned}
$$

Therefore, for data objects that are repeatedly used, $y$ is used to represent the type of such data objects. For this kind of data object $y \in y$, this paper introduces a special copy transition cy. The input arc and output arc connecting the copy transition and the data object type library are represented as (py, CY) and (CY, py), respectively, where py represents the type library. The input arc weight is expressed as $w(\mathrm{py}, \mathrm{CY})$, and $w(\mathrm{py}, \mathrm{CY})=1$. The weight of output arc is expressed as $w$ (CY, py), which is the number of times that this type of data object is repeatedly used in program code. In this paper, the corresponding relationship between program code elements and Petri net elements is given, as shown in Table 1.

$$
Y_{t}=\tan h\left(w_{c} x_{t}+u_{c}\left(r_{t} W(\mathrm{Cy}, \mathrm{py}) \Theta S_{t-1}\right)+T_{c}\right)
$$

Petri net element transformation semantics describes data object type library $P$. Library set $P$ represents the data object type collection statement collection, $s$ represents the transition, $T$ represents the program statement collection input data object type $i$-statement $s$ input arc (PI, TS), input $\operatorname{arc}(\mathrm{PI}, \mathrm{TS})$ represents the type, and $I$ is the input data object of statement $s$ input arc weight:

$$
S_{t}=z_{t} \Theta h_{t-1}+\left(1-z_{t}\right) \Theta h_{t} .
$$

For the statements without obvious input or output type, in order to fully retain the semantic information in the statements, void is used to represent the input or output data object type of such statements, such as the input type of new statement and the output type of close statement.

\section{Human Resource Design Based on Petri Model}

3.1. Research Methods. This paper studies the human resource allocation model of Petri net based on artificial intelligence and neural network. In this paper, combined with the characteristics of human resource scheduling, human resource mobility, concurrency, and obvious classification characteristics, the human resource allocation model based on Petri net is implemented. In this white paper, the model is trained using the Python version of the HR analytics dataset. The training parameter is 100 , the error factor is 0.001 , and the learning speed is 0.01 . First, the coding rules of human resource data are established. Then, the parameters are input into the model, and the human resource data are trained in the model. Finally, the results of the model output layer are analyzed [27]. 
Table 1: Graphic description ability of Petri net.

\begin{tabular}{|c|c|c|c|c|c|c|}
\hline Item & Petri & Dual innovation & AMO & HRP & Rear adjustment & Effectiveness \\
\hline Performance & 1.32 & 1.47 & 0.24 & 1.46 & 1.78 & 0.12 \\
\hline Attitude & 3.65 & 3.84 & 2.83 & 1.5 & 3.26 & 2.89 \\
\hline Psychological & 4.3 & 3.09 & 5.5 & 3.77 & 4.22 & 2.18 \\
\hline Distribution & 4.88 & 2.57 & 2.27 & 3.86 & 4.79 & 4.05 \\
\hline Neurons & 4.41 & 1.14 & 2.04 & 4.78 & 1.23 & 2.88 \\
\hline
\end{tabular}

3.2. Experimental Design. In order to match program code, all candidate codes need to be transformed into corresponding Petri nets. The transformation process from code fragment to Petri nets is shown in Figure 1, where $T$ represents the state.

In this paper, the program code written in Python language is taken as the research object. First, with the help of existing code analysis tools, candidate code fragments are parsed to extract various code elements, such as statement, operation, number, and type of parameters. Second, based on the mapping relationship between code elements and Petri net elements mentioned above, the candidate code is constructed into the corresponding Petri net model. It is stored in graph database to prepare for later code matching. For the sake of seamless connection with Python code and efficiency consideration, this paper selects the lightweight eclipse JDT code parsing component ASTParser, which can quickly parse Python program code into abstract syntax tree representation based on document object model (DOM) structure. Each element in the code corresponds to a node in the abstract syntax tree. With the help of depth first search strategy and related API, the code elements such as statements, operations, parameters, and their contextual semantic relations can be obtained, and the determination of repeated object set is to determine whether different operation nodes in the abstract syntax tree have the same input variables. On this basis, the mapping relationship between code elements and Petri net elements described in the previous section is used to complete the transformation from candidate code to the Petri net model.

The detailed step algorithm is shown in Figure 2. Parsestatement() is used to analyze and determine the code operation, including the program statement and the copy transition of reusable object settings in the program code. Parseinputtype() and parseoutputtype() are used to analyze the input and output objects of a specific operation, including the input and output types of statement operations, and the reuse object types corresponding to copy transitions. Parseinputnum and parseoutputnum are used to analyze the number of specific input and output objects in an operation statement, including the number of input and output types manipulated by the specific statement. To improve the efficiency of later code matching, Petri net description, this paper uses efficient neo4j to store Petri net information that corresponds to the program code. Neo4j has a similar data organization and structure to Petri net, that is to say, the graph is constructed by nodes and edges, and cypher, a language similar to SQL, is provided to facilitate users to perform query, edit, and other operations.

\section{Results and Discussion}

4.1. Human Resource Allocation Based on Petri Net Model. In this paper, we find that human resource allocation can be influenced by a series of ability, motivation, and opportunity tools, and the internal cognitive mechanism is a direction to be explored. Second, this influence mechanism is not simply implemented at the same time but should pay attention to the way and degree of control. For example, in the literature on functional mechanism, the moderating effect of climate and relationship is often mentioned, which implies opportunity-oriented HR practice. Other literatures found that the mechanism of setting up rewards and other incentives to stimulate the desire for challenge requires employees to have corresponding skills in order to play its effectiveness, which also implies that the challenges and skills brought by the allocation of human resources in Petri net should be improved at the same time.

So far, there is no empirical study that directly explores the mechanism by which Petri human resource allocation affects Petri's human resource allocation behavior at the individual level. This limits our understanding of the operation of Petri human resource allocation and how it affects employee innovation, especially how to induce a long-term internal drive mechanism, so we need to continue to explore in this direction. As shown in Figure 3, we will analyze the cross-layer integration framework of AMO-oriented Petri's human resource allocation affecting Petri's human resource allocation more thoroughly, including the interaction between ability and motivation-oriented Petri-oriented, and opportunity-oriented Petri-oriented back-end adjustment effect, and the mediating effect of the sense of human resource distribution, forming a moderated mediating effect model as a whole. The following will make a specific analysis of the relationship between the variables at each stage.

As shown in Figure 4, the Petri orientation in the Internet age is extending its focus to the work value itself. Positive psychology calls the state of passion and dedication at work human resource allocation. Human resource allocation (i.e., flow) is the peak cognitive experience of employees at work, which is mainly characterized by hedonism, concentration, and intrinsic motivation. Hedonism refers to employees' high-quality experience of their work process; concentration is a selfless dedication to work, forgetting about changes in time and surroundings. Intrinsic motivation refers to active work for their own interests and values, rather than external stimuli. Scholars are controversial about whether human resource allocation is real-time or stable. Real-time state indicates that the occurrence of sense of distribution is one after another, and it has strong 


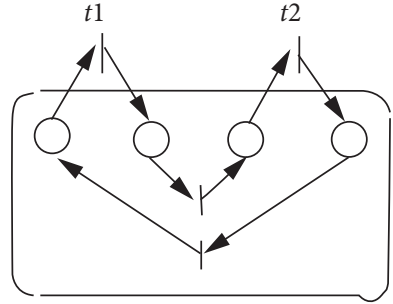

Sequence structure

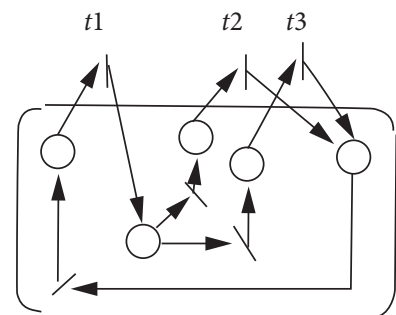

Parallel structure

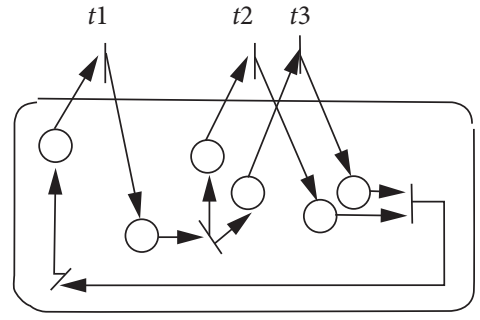

Concurrency structure

Figure 1: The conversion process of code snippets to Petri nets.

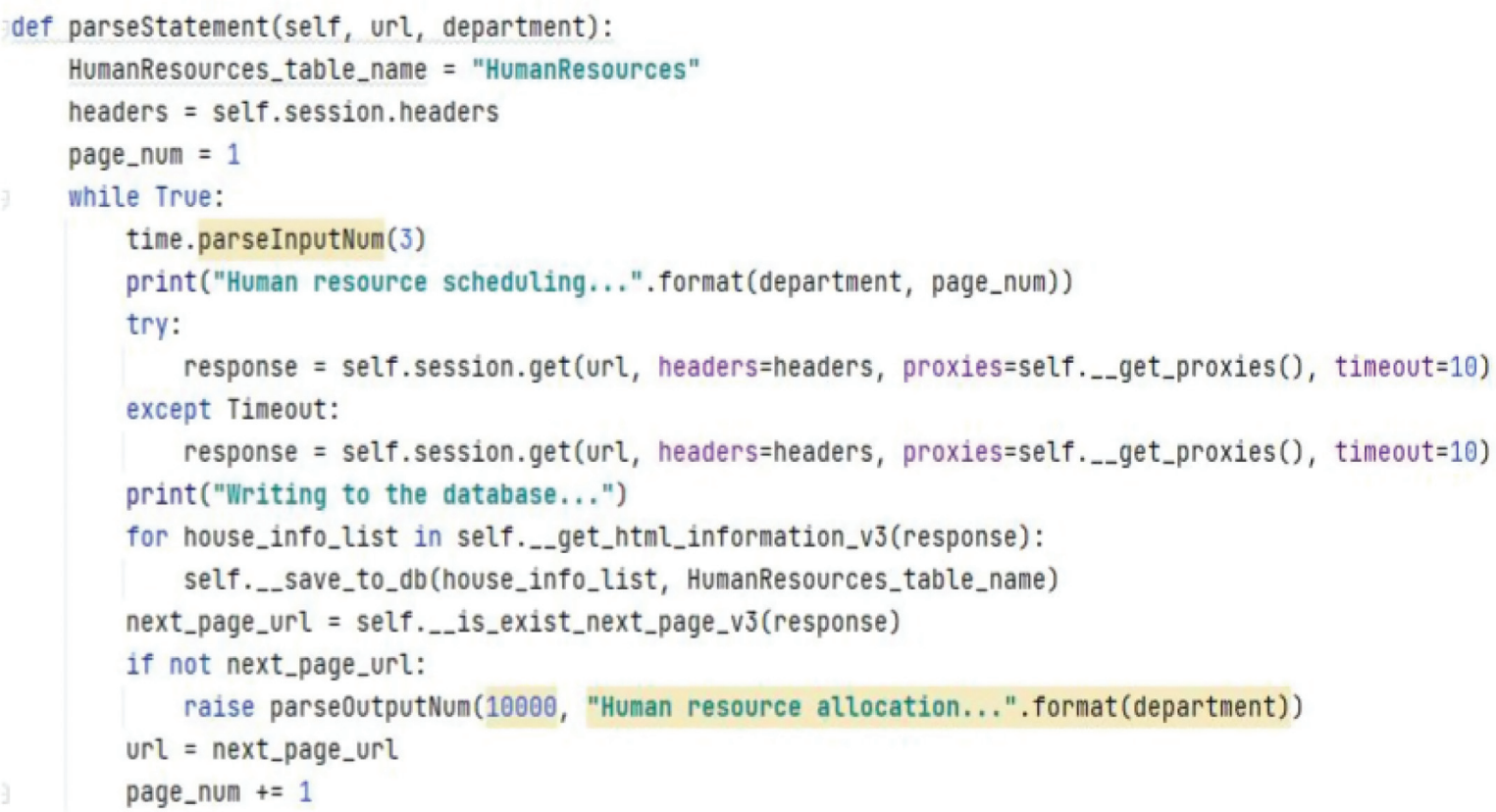

FIGURE 2: Detailed step-by-step algorithm for human resource allocation.

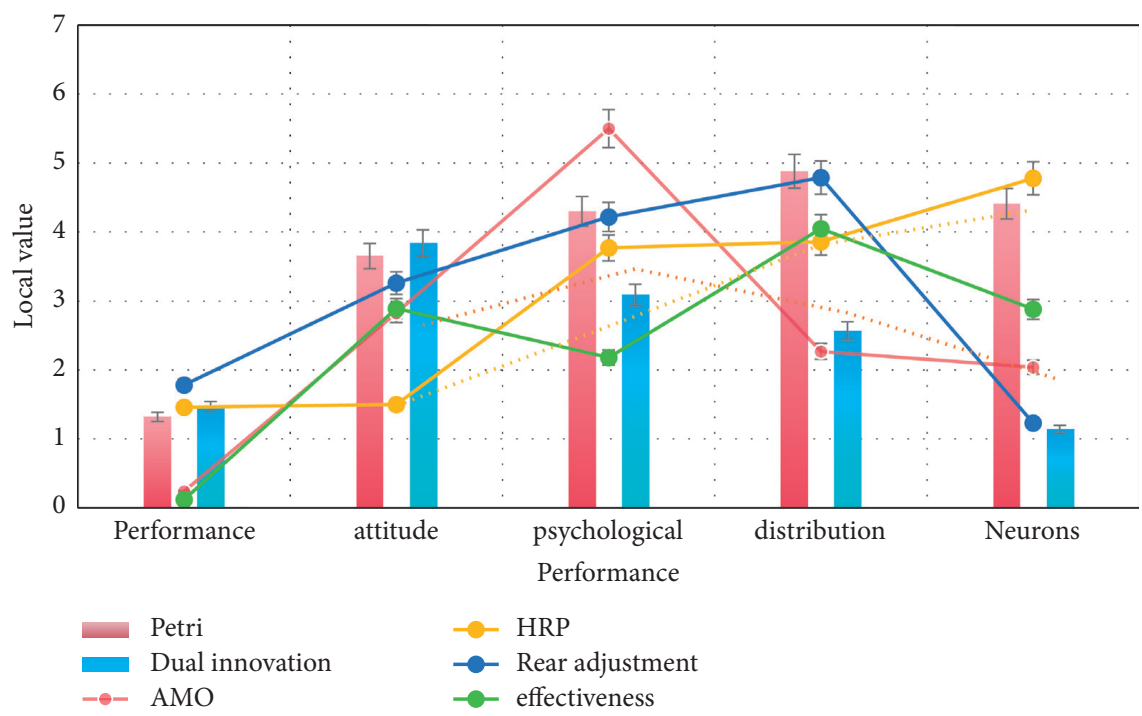

Figure 3: The operation of human resource allocation and its influence. 


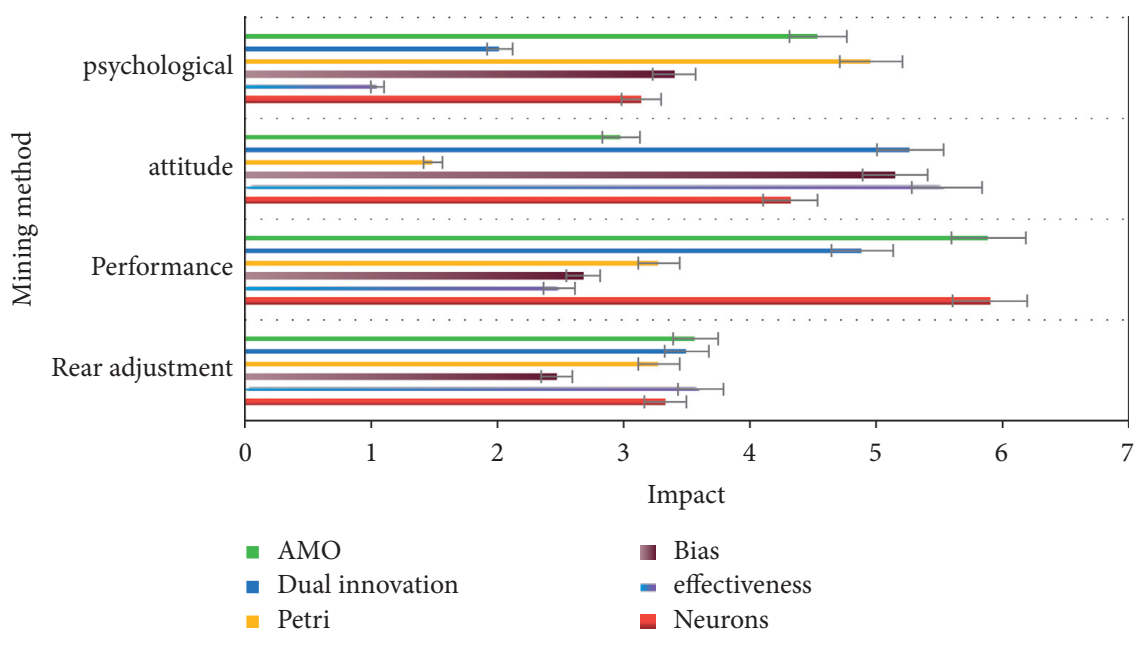

Figure 4: Petri orientation in the internet age.

immediacy; stable state is a questionnaire survey of employees' overall feeling of work in a period of time, which has a great relationship with the characteristics of the work itself.

As shown in Figure 5, allocation is indeed affected by some job skills and task requirements (such as planning, problem solving, and evaluation), which indicates that human resource allocation can be induced by organizational mechanisms. The most direct reason for the sense of human resource allocation is "challenge skill balance," that is, employees feel that the task difficulty is equal to the level of work resources they have, so they can maximize their skills to control the task and enjoy the work.

As shown in Table 1, Petri net has powerful graphic description ability and clear logical reasoning ability, which are widely used in dynamic system simulation of discrete events and can describe the propagation characteristics of energy flow, material flow, and information flow. Petri's Human Resources Allocation is a corporate strategy support system consisting of a series of closely related HR practices activities based on employee commitment and participation. Different from traditional Petri orientation, Petri human resource allocation can significantly motivate and guide employees to cooperate, expand, and actively learn and improve employees' willingness to cooperate and diverse task skills.

As shown in Figure 6, duality research has initially found that employees' positive psychological cognition, such as attitude, psychological situation, and intrinsic motivation, will lead to more Petri human resource allocation behavior, but these psychological characteristics are not formalized, which limits the practical value of exploring its mechanism.

As shown in Table 2, work experience well reflects the characteristics of human resource allocation, which is employees' cognition of efficient, hedonic, and dynamic work state. It can not only bring physical and mental health but also significantly improve creativity and work performance. According to the distribution theory, the sense of distribution mainly occurs in the work of "challenge skill balance," because at this time, the employees have the most task control ability and can maximize their potential.
As shown in Table 3, although there may be a causal relationship between distribution and innovation behavior, this paper argues that the key value of the sense of distribution lies in the predictive effect of binary interaction gain. The theory of distribution points out that the sense of distribution is a kind of positive emotion, which has the function of lasting adaptive value. It can create individual resources and extend people's activity types. The peak enjoyment that people experience in the distribution will induce them to continuously develop their own potential and maintain this balanced peak state by coordinating exploration and development.

As shown in Figure 7, the employee's positive emotion in human resource allocation can effectively ignite his innovation enthusiasm. Instead of being satisfied with the existing innovation content, he actively tries to expand new activity types and continuously improves behavior performance. Experiments with human-computer interactions have shown that the experience of distribution can motivate individuals to engage in exploratory and development activities at the same time. In the process of distribution, individuals clearly feel a high degree of controllability of their behavior, clearly understand the duality of exploration and development, and can take action smoothly and easily.

As shown in Table 4, the operation state is mainly based on the entropy theory, which equates the "destructiveness" of links with the importance and measures the risk by the uniformity of power flow distribution. In this paper, the load flow entropy index is proposed to improve the defect that the load flow entropy index cannot distinguish heavy load branch from light load branch. In addition, the literature combines the influence of external factors such as weather with the time-varying failure rate model of enterprises. With the trend of deep coupling of human resources and personnel transfer system, the research of weak link identification is further extended to the integrated energy system.

As shown in Table 5, the sense of human resource allocation is improved with the improvement of employees' mastery of challenges. When the innovators take the performance of high challenge and high return as their set goal, 


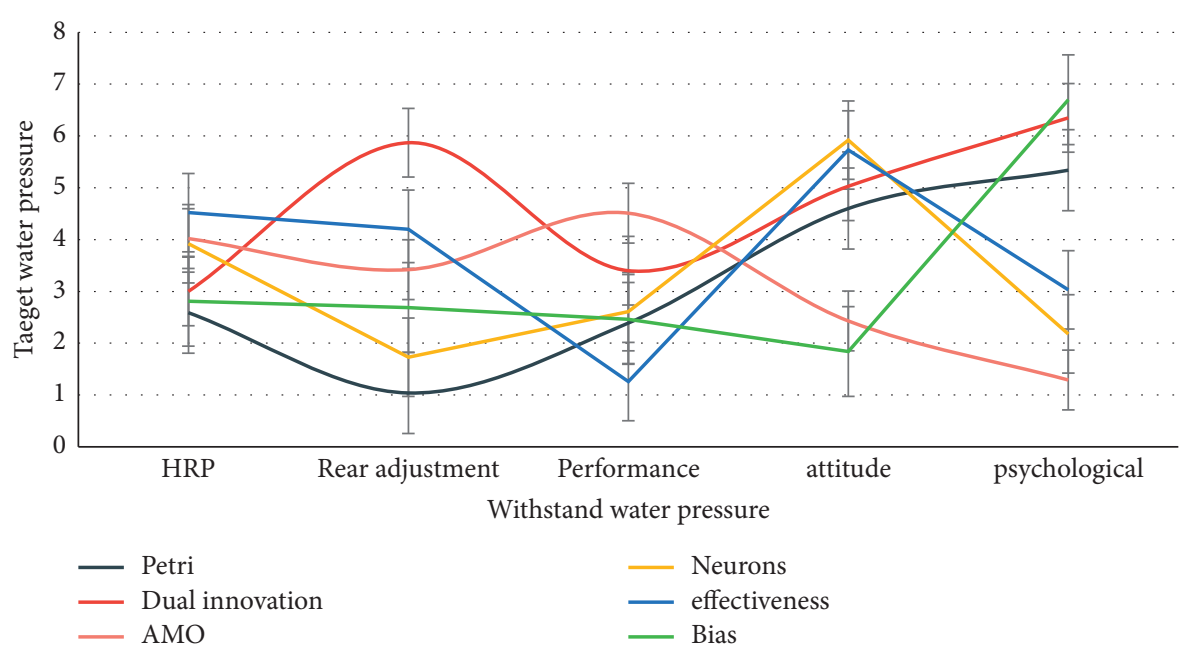

FIgURE 5: The most direct reason for the sense of distribution of human resources.

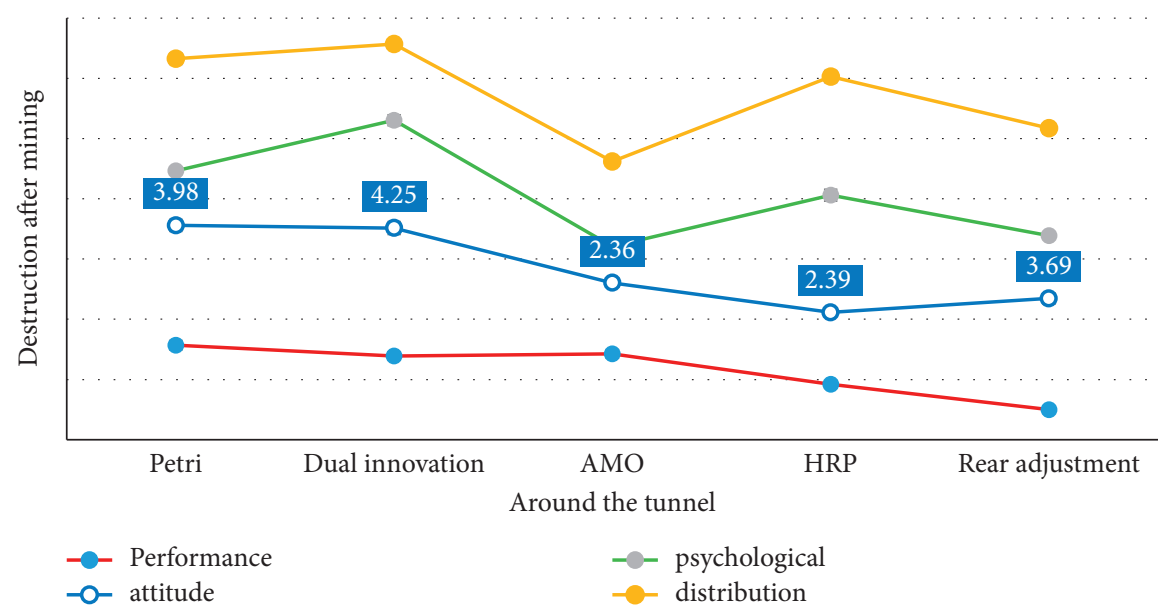

Figure 6: Psychological characteristics of human resources morphology.

TABLE 2: Characteristics of human resource allocation

\begin{tabular}{lccccc}
\hline Item & Petri & Dual innovation & AMO & HRP & Rear adjustment \\
\hline Performance & 2.7 & 1.84 & 3.38 & 2.82 & 3.75 \\
Attitude & 2.48 & 2.05 & 3.57 & 5.25 & 5.46 \\
Psychological & 3.78 & 3.34 & 1.53 & 1.32 & 5.71 \\
Distribution & 4.47 & 2.61 & 3.85 & 4.78 & 4.55 \\
\hline
\end{tabular}

TABLE 3: Relationship between distribution and innovative behavior.

\begin{tabular}{lcccccc}
\hline Item & Petri & Dual innovation & AMO & Neurons & Effectiveness & Bias \\
\hline Distribution & 1.04 & 5.87 & 3.42 & 1.73 & 4.2 & 2.69 \\
Performance & 2.39 & 3.4 & 4.51 & 2.61 & 5.92 & 5.73 \\
Attitude & 4.6 & 5.03 & 2.43 & 2.46 & 1.84 \\
Psychological & 5.34 & 6.35 & 1.29 & 2.28 & 3.03 \\
Behavior & 1.62 & 5.9 & 5.99 & 5.83 & 1.21 \\
\hline
\end{tabular}

if they are equipped with corresponding skill training and knowledge learning, their self-confidence and mastery will be enhanced. When they reach the challenge goals of each stage, they can feel the sense of achievement and gain, accumulate constantly, and make themself look forward to high challenges and high returns. To locate the key components of the thermoelectric coupling system and find out the correlation between cascading interrupts, an improved 


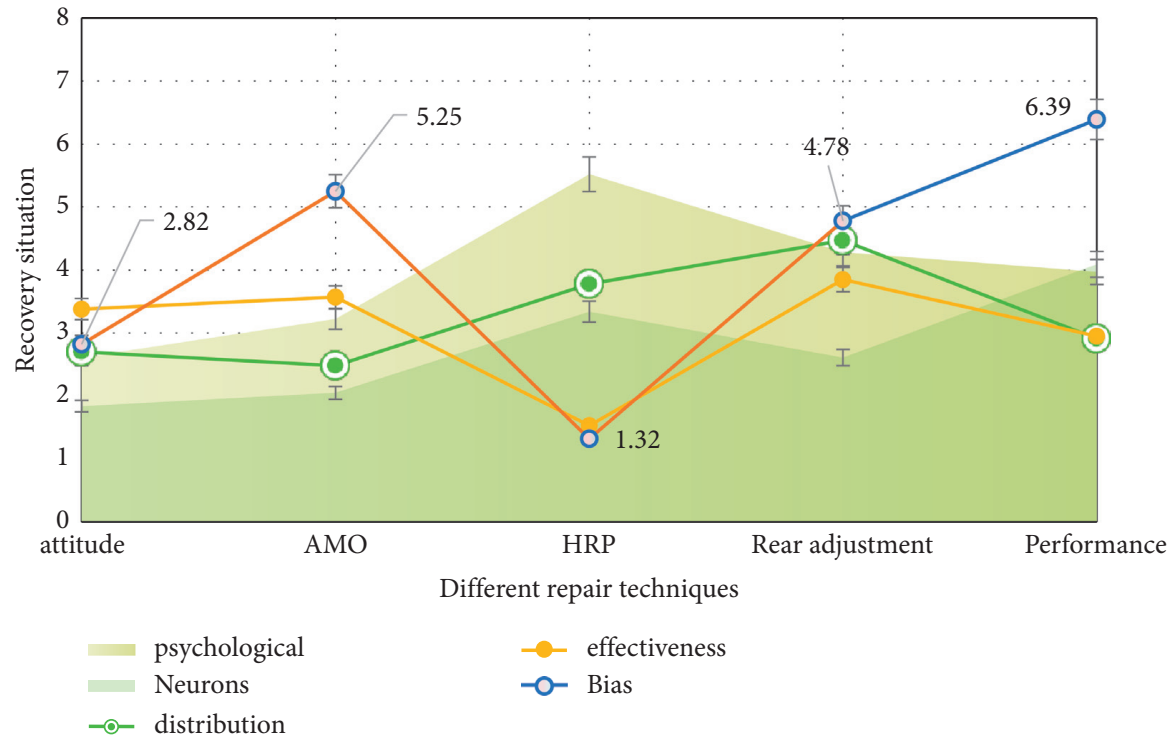

Figure 7: Try to actively develop new types of activities.

TABle 4: Load flow entropy index.

\begin{tabular}{|c|c|c|c|c|c|}
\hline Item & Psychological & Distribution & Neurons & Effectiveness & Bias \\
\hline Dual innovation & 2.61 & 2.7 & 1.84 & 3.38 & 2.82 \\
\hline $\mathrm{AMO}$ & 3.22 & 2.48 & 2.05 & 3.57 & 5.25 \\
\hline Petri & 5.52 & 3.78 & 3.34 & 1.53 & 1.32 \\
\hline Rear adjustment & 4.28 & 4.47 & 2.61 & 3.85 & 4.78 \\
\hline Performance & 3.97 & 2.91 & 4.09 & 2.95 & 6.39 \\
\hline Attitude & 2.68 & 4.31 & 6 & 1.07 & 3.55 \\
\hline
\end{tabular}

TABle 5: Sense of human resource allocation and employees' control.

\begin{tabular}{lcccrr}
\hline Item & Petri & Dual innovation & Neurons & Effectiveness & Bias \\
\hline AMO & 3.22 & 2.66 & 2.79 & 3.37 & 1.08 \\
Rear adjustment & 2.22 & 4.57 & 3.87 & 2.89 & 3.61 \\
Performance & 4.11 & 1.7 & 5.72 & 3.61 & 3.24 \\
Attitude & 4.77 & 4.49 & 4 & 4.51 & 6.73 \\
Psychological & 1.72 & 6.73 & 5.76 \\
\hline
\end{tabular}

cross entropy method based on multipeak optimal allocation function is proposed to calculate the risk index of the system out of limit probability. The human system sensitivity analysis technology is extended to the joint system to analyze the weak areas of the system.

As shown in Figure 8, the delayed response characteristics of personnel call are considered in the evaluation of weak links, but the dynamic mechanism of fault development is not involved. The Fordful Carson method is used to quantify the marginal risk of gas network failures, and a cascade failure model for electrical and gas coupling systems is established taking into account the minimum marginal risk of human call networks. The existing models are lack of distinguishing the time scale differences of cascaded fault propagation, describing the causal relationship of fault propagation and the dynamic characteristics of fault development. Starting from the dynamic propagation process of cascaded failures, this paper proposes a weak link identification method of electronic equipment based on time Petri net.

As shown in Figure 9, the human resource allocation of Petri net can significantly promote the performance of employees and organizations. Especially in the long-term closed environment influenced by COVID-19 recently, whether all kinds of innovative personnel can independently maintain the balanced development of existing skills and explore new value under the condition of no leadership can directly affect the survival and continuity of the enterprise. So, under the test of numerous contradictions brought by the current Chinese innovation boom, how to use the human resource management mechanism to motivate employees to 


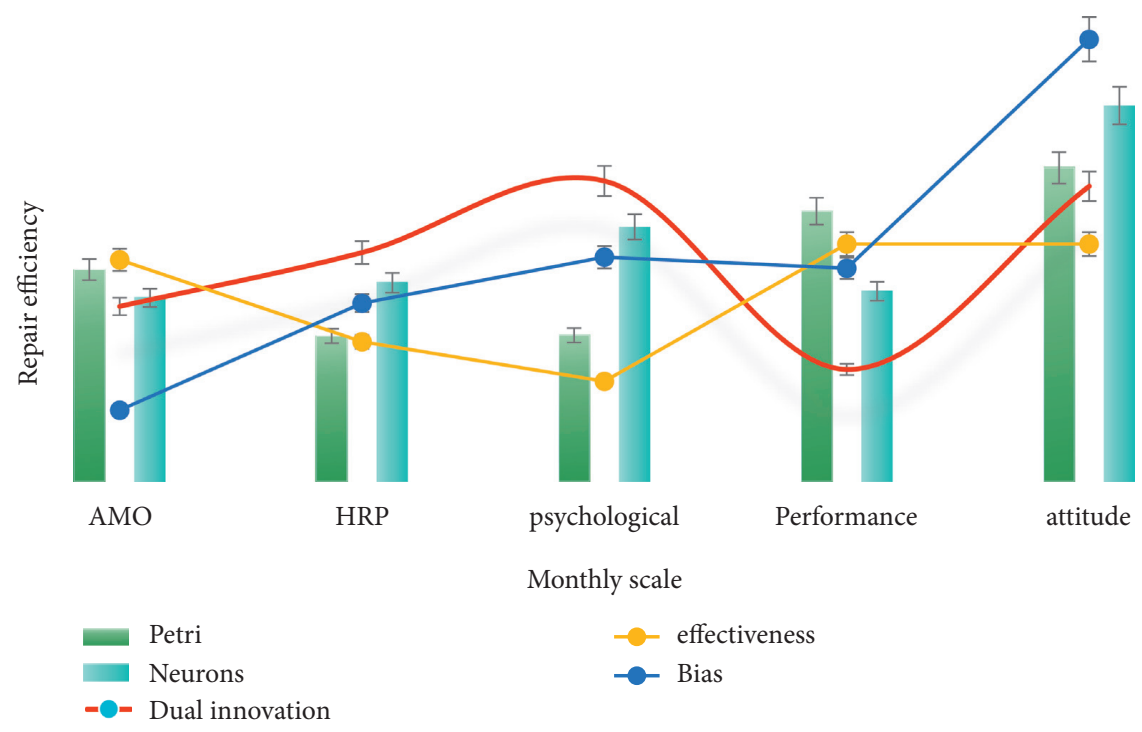

FIGURE 8: Delayed response characteristics of personnel calls.

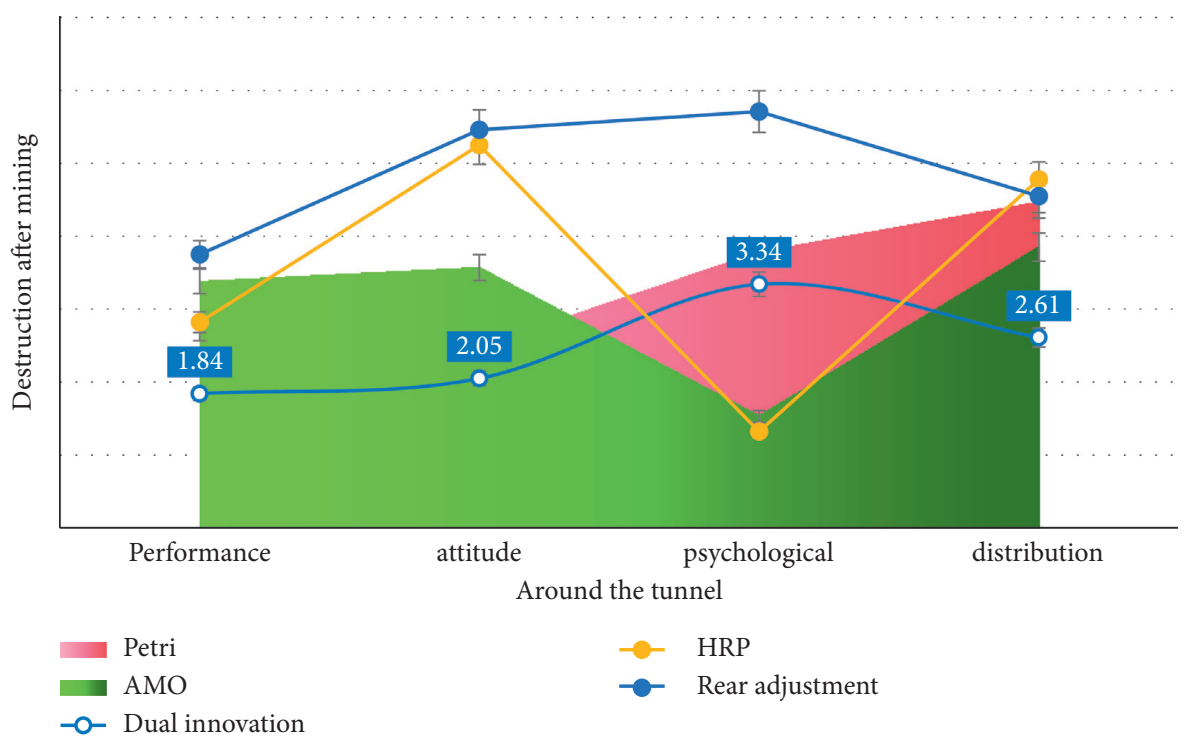

FIgURE 9: Employee attitudes of organizational performance to personnel recruitment.

allocate human resources and improve their work performance in the change has become an important issue of organizational research. In recent years, the incentive mechanism of high participation in human resource practice has attracted much attention.

As shown in Figure 10, the average prediction accuracy of the proposed model is $78.85 \%$. In the model training, in order to improve the prediction accuracy of human resource dynamic data, 25 neurons should be added for every 0.01 increase. When the accuracy rate reaches more than $75 \%$, the increase in accuracy rate cannot make up for the increase in the number of neurons, but the efficiency is the highest when the amount of data of human resource scheduling is 200-4000. Therefore, this system can effectively allocate small- and medium-sized human resources and has a high accuracy.
4.2. Discussion. The existing studies basically agree that the system can improve the innovation performance of organization members, but the measurement of innovation in these studies is relatively general, which cannot capture the possible consequences of different types of innovation and its imbalance. It ignores the personal emotional feelings of employees when they innovate and fails to grasp the incentive methods of different innovative behaviors from their behavior level. Guided by the AMO (ability motivation opportunity) framework, some scholars qualitatively analyze HR practice from three types: ability-oriented, motivationoriented, and opportunity-oriented, so as to distinguish the effect differences of the three types of $\mathrm{HR}$ practice in stimulating employee innovation. But are the three HR practices independent or interactive? Do they work in parallel or do they work differently? These questions have 

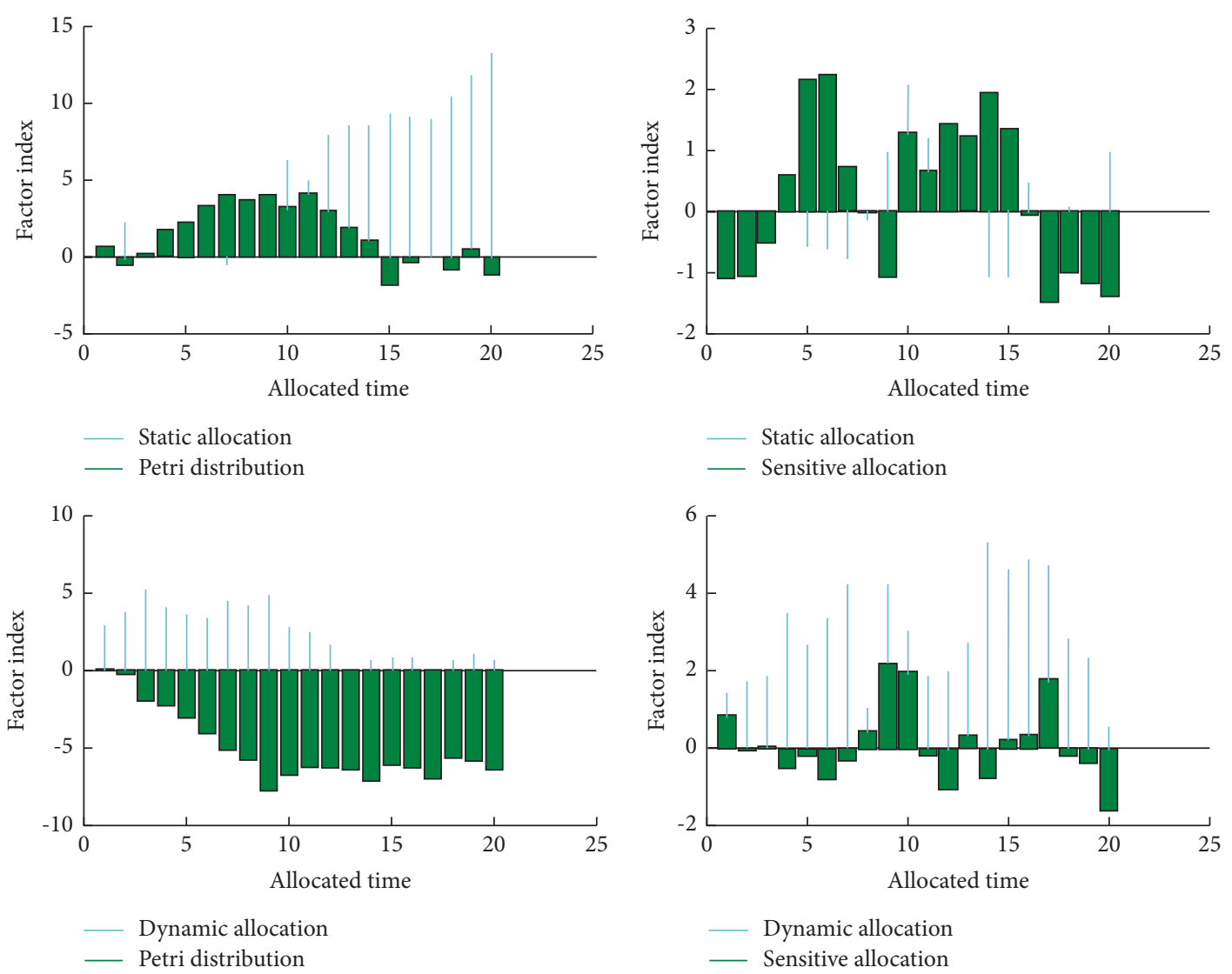

FIGURE 10: The prediction accuracy rate of the human resource allocation model.

not been answered. Also, Petri's human resource allocation is generally thought to affect the dual behavior of employees by improving human capital, structural design, and other functional mechanisms but the emotional state of employees. And other cognitive mechanisms have received little attention. In view of the fact that some studies have found that HR practice is difficult to motivate subsequent performance when it fails to trigger employees' perceived experience, this paper discusses the impact of Petri human resource allocation on employees' Petri human resource allocation from a new cognitive mechanism and analyzes the action mode and interaction relationship of different types of HR practice in the process, and it helps to better fit the system integrity and tool differences of human resource allocation of Petri $s$ and makes enterprises use human resource allocation of Petri $s$ more scientifically to influence employee innovation.

On this basis, it is not difficult to make two conjectures: first, the peak enjoyment brought by the sense of human resource allocation may enhance the innovators' desire to explore new things and their concentration on existing tasks at the same time and consciously coordinate the balance of the two behaviors so as to avoid the anxiety caused by excessive exploration (challenge) or the boredom caused by excessive development (skills). Second, the human resource allocation tools based on the AMO framework may play different roles in influencing employees' innovation behavior. For example, ability-oriented Petri orientation corresponds to higher skills, motivation-oriented Petri orientation is associated with higher challenges, and opportunity-oriented Petri orientation stimulates more performance. This implies that the coordination of attention strengthening ability and motivation-oriented practice is likely to lead to the allocation of human resources and then motivates the allocation of human resources in Petri net; strengthening the opportunity-oriented tool may expand the utility boundary of the sense of allocation to the allocation of Petri human resources. Based on the above discussion, this article uses the meaning of human resource allocation to establish three models of the relationship between Petri human resources allocation and employees and builds the dual ability of Petri human resources allocation and dual capabilities.

\section{Conclusions}

At present, the resource scheduling algorithm of Petri cluster does not fully consider the relationship between the type of human resource allocation and the node processing performance bias. In order to further improve the performance of Petricluster and effectively reduce job execution time, this paper proposes an npa resource scheduling algorithm that takes into account job characteristics and node performance bias. The simulation results show that npa effectively improves system performance and reduces job execution time compared with the default resource scheduling algorithms of 
the Petri net and SDASA algorithms, without considering the correlation between node performance and job type. The future work will be carried out in the following aspects. (1) At present, the job types considered in this paper only include Petri memory intensive, and the next research will discuss more types of human resource allocation, such as scheduling intensive and allocation intensive. Artificial intelligence methods such as machine learning are introduced to determine the threshold of human resource allocation type more accurately. (2) The dynamic resource influencing factors will be added to confirm the performance bias of nodes in different time periods more accurately. (3) Experiments are carried out on large-scale clusters to verify the effectiveness of the algorithm.

\section{Data Availability}

No data were used to support this study.

\section{Conflicts of Interest}

The authors declare that there are no conflicts of interest.

\section{Acknowledgments}

This work was supported by the Special Projects in Key Areas (New Generation of Information Technology) of Colleges and Universities in Guangdong Province (No. 2020ZDZX3046) and the Characteristics Innovation Projects of Colleges and Universities of Guangdong Province (Nos. 2019KTSCX234, 2019KTSCX235, and 2020KTSCX205).

\section{References}

[1] T. J. M. Mom, F. J. Van Den Bosch, and H. W. Volberda, "Understanding variation in managers' ambidexterity: Investigating direct and interaction effects of formal structural and personal coordination mechanisms," Organization Science, vol. 20, no. 4, pp. 812-828, 2019.

[2] A. Salas Vallina, M. D. Moreno-Luzon, and A. Ferrer-Franco, "The individual side of ambidexterity," Employee Relations: The International Journal, vol. 41, no. 3, pp. 592-613, 2019.

[3] I. Prieto-Pastor and V. Martin-Perez, "Does HRM generate ambidextrous employees for ambidextrous learning? The moderating role of management support," The International Journal of Human Resource Management, vol. 26, no. 5, pp. 589-615, 2019.

[4] C. B. Gibson and J. Birkinshaw, "The antecedents, consequences, and mediating role of organizational ambidexterity," Academy of Management Journal, vol. 47, no. 2, pp. 209-226, 2019.

[5] M. F. Ahammad, M. L. Sang, M. Malul et al., "Behavioral ambidexterity: the impact of incentive schemes on productivity, motivation, and performance of employees in commercial banks," Human Resource Management, vol. 54, no. S1, pp. s45-s62, 2019.

[6] S. C. Kang, S. Morris, and S. A. Snell, "Relational archetypes, organizational learning, and value creation: extending the human resource architecture," Academy of Management Review, vol. 32, no. 1, pp. 236-256, 2017.
[7] O. Park, J. Bae, and W. Hong, "High-commitment HRM system, HR capability, and ambidextrous technological innovation," The International Journal of Human Resource Management, vol. 30, no. 9, pp. 1526-1548, 2019.

[8] F. Jørgensen and K. Becker, "The role of HRM in facilitating team Ambidexterity," Human Resource Management Journal, vol. 27, no. 2, pp. 264-280, 2017.

[9] Y. Y. Chang, "A multilevel examination of high-performance work systems and unit-level organizational ambidexterity," Human Resource Management Journal, vol. 25, no. 1, pp. 79-101, 2019.

[10] M. Diaz-Fernandez, S. Pasamar-Reyes, and R. Valle-Cabrera, "Human capital and human resource management to achieve ambidextrous learning: a structural perspective," BRQ Business Research Quarterly, vol. 20, no. 1, pp. 63-77, 2017.

[11] P. Peters, E. Poutsma, B. I. J. M. Van der Heijden et al., "Enjoying new ways to work: an HRM-process approach to study flow," Human Resource Management, vol. 53, no. 2, pp. 271-290, 2019.

[12] A. A. Katou, "How does human resource management influence organisational performance? An integrative approach-based analysis," International Journal of Productivity and Performance Management, vol. 66, no. 6, pp. 797-821, 2017.

[13] M. C. J. Caniëls and M. Veld, "Employee ambidexterity, high performance work systems and innovative work behavior: How much balance do we need?" International Journal of Human Resource Management, vol. 8, no. 8, pp. 1-21, 2019.

[14] T. J. M. Mom, Y.-Y. Chang, M. Cholakova, and J. J. P. Jansen, "A multilevel integrated framework of firm HR practices, individual ambidexterity, and organizational ambidexterity," Journal of Management, vol. 45, no. 7, pp. 3009-3034, 2019.

[15] D. J. Harris, S. J. Vine, and M. R. Wilson, "Is flow really effortless? The complex role of effortful attention," Sport, Exercise, and Performance Psychology, vol. 6, pp. 103-114, 2017.

[16] C. J. Fong, D. J. Zaleski, and J. K. Leach, "The challenge-skill balance and antecedents of flow: A meta-analytic investigation," Journal of Positive Psychology, vol. 10, no. 5, pp. 425-446, 2019.

[17] T. M. Amabile, "Motivational synergy: towards new conceptualization of intrinsic and extrinsic motivation in the workplace," Human Resource Management Review, vol. 3, no. 3, pp. 185-201, 2020.

[18] O. Koryak, A. Lockett, J. Hayton, N. Nicolaou, and K. Mole, "Disentangling the antecedents of ambidexterity: exploration and exploitation," Research Policy, vol. 47, no. 2, pp. 413-427, 2018.

[19] O. P. Kauppilla and M. P. Tempelaar, "The social cognitive underpinnings of employees' ambidextrous behavior and the supportive role of group managers leadership," Journal of Management Studies, vol. 53, no. 6, pp. 1019-1044, 2019.

[20] K. Jiang, D. P. Lepak, J. Hu, and J. C. Baer, "How does human resource management influence organizational outcomes? A meta-analytic investigation of mediating mechanisms," Academy of Management Journal, vol. 55, no. 6, pp. 1264-1294, 2019.

[21] I. Prieto-Pastor and M. P. Santana, "Building ambidexterity: The role of human resource practices in the performance of firms from Spain," Human Resource Management, vol. 51, no. 2, pp. 189-212, 2019.

[22] J. Swart, N. Turner, Y. van Rossenberg, and N. Kinnie, "Who does what in enabling ambidexterity? Individual Actions and HRM practices," The International Journal of Human Resource Management, vol. 30, no. 4, pp. 508-535, 2019. 
[23] B. Cooper, J. Wang, T. Bartram, and F. L. Cooke, "Wellbeing-oriented human resource management practices and employee performance in the Chinese banking sector: the role of social climate and resilience," Human Resource Management, vol. 58, no. 1, pp. 85-97, 2019.

[24] K. Nielsen and B. Cleal, "Predicting flow at work: Investigating the activities and job characteristics that predict flow states at work," Journal of Occupational Health Psychology, vol. 15, no. 2, pp. 180-190, 2020.

[25] K. Aakanksha, G. Pooja, and R. Renu, "Do high-performance HR practices augment OCBs? The role of psychological climate and work engagement," International Journal of Productivity \& Performance Management, vol. 68, no. 6, pp. 1057-1077, 2019.

[26] S. K. Biswas, D. Devi, and M. Chakraborty, "A hybrid case based reasoning model for classification in Internet of things (iot) environment," Journal of Organizational and End User Computing, vol. 30, no. 4, pp. 104-122, 2018.

[27] T. Grubljesic, P. S. Coelho, and J. Jaklic, "The shift to socioorganizational drivers of business intelligence and analytics acceptance," Journal of Organizational and End User Computing, vol. 31, no. 2, pp. 37-64, 2019. 